\title{
Diagnósticos de enfermagem e proposta de intervenções para pacientes com lesão medular
}

\author{
Nursing diagnoses and interventions proposal al for patients with spinal cord lesion
}

\author{
Diagnósticos de enfermería y propuesta de intervenciones \\ para pacientes con lesión medular
}

\author{
Clélia Regina Cafer ${ }^{1}$, Alba Lucia Bottura Leite de Barros², Amália de Fátima Lucena ${ }^{3}$, \\ Maria de Lourdes Sylvestre Mahl ${ }^{4}$, Jeanne Liliane Marlene Michel ${ }^{5}$
}

\section{RESUMO}

Introdução: As lesões medulares constituem evento grave que acomete principalmente jovens, requerendo um planejamento cuidadoso da assistência de enfermagem Este estudo tem a finalidade de contribuir nesse planejamento, oferecendo subsídios para melhorar a qualidade do cuidado desses pacientes. Objetivos: Identificar os diagnósticos de enfermagem, segundo a NANDA, em portadores de lesão medular internados numa Unidade de Ortopedia e Traumatologia; e propor as intervenções de enfermagem, conforme a NIC. Métodos: Estudo descritivo prospectivo, com 10 casos analisados no período de janeiro/2000 a julho/2002. Os dados foram coletados por meio de um instrumento criado para uso na unidade de estudo, identificando-se os diagnósticos de enfermagem. As intervenções foram propostas pelas autoras e submetidas à avaliação de duas especialistas. Resultados: Foram identificados 15 diagnósticos de enfermagem prevalentes, para os quais foram propostas 26 intervenções de enfermagem. Conclusão: Os resultados poderão subsidiar as enfermeiras ao cuidado do paciente com lesão medular, auxiliando-o e contribuindo à sua autonomia, através das intervenções propostas.

Descritores: Diagnóstico de enfermagem; Traumatismos da coluna vertebral; Cuidados de enfermagem

\section{ABSTRACT}

Introduction: Spinal cord lesions constitute very serious events that occur mostly among young people. Providing care to these patients is a complex task because they are greatly dependent on nursing staff and require a very carefully designed plan of care. The purpose of this study was to contribute ways to design a quality plan of care to attend these patients. Objectives: To identify nursing diagnoses according to the NANDA for patients with spinal cord lesions from an Orthopedic and Trauma Unit, and to propose appropriate nursing interventions according to the NIC. Methods: This study used a descriptive and prospective research design. The sample consisted of 10 patients with spinal cord lesions. Data were colleted from January, 2000 to July, 2002 using a specific assessment tool. Data analysis led the researchers to propose nursing diagnoses which were validated by two clinical nursing specialists. Results: Fifteen prevalent nursing diagnoses were identified and 26 nursing interventions were proposed. Conclusion: The results can be used to assist nurse to provide quality care for patients with spinal cord lesions, so as to improve the patients independence.

Keywords: Nursing diagnosis; Spinal injuries; Nursing care

\section{RESUMEN}

Introducción: Las lesiones medulares constituyen un evento grave que afecta, en su mayoría, a los jóvenes, las cuales requirien una planificación cuidadosa para la atencion de enfermeria. Este estudio tiene la finalidad de contribuir con subsidios para mejorar la calidad de los cuidados prestados a esos pacientes. Objetivos: Identificar los diagnósticos de enfermería, según la taxonomía de la NANDA, en pacientes con lesión medular, internados en una Unidad de Ortopedia y Traumatología; y proponer las intervenciones de enfermería, conforme el referencial de la NIC. Métodos: Estudio descriptivo prospectivo en el cual fue analizado un total de 10 casos, en el período de enero/2000 a julio/2002. Los datos fueron recolectados por medio de un instrumento creado para uso en la unidad de estudio, con la finalidad de identificar los diagnósticos de enfermería. Las intervenciones fueron propuestas por las autoras y sometidas a la evaluación de dos especialistas. Resultados: Fueron identificados 15 diagnósticos de enfermería prevalentes, para los cuales se propusieron 26 intervenciones de enfermería. Conclusión: Los resultados del estudio servirán de subsidio a las enfermeras para el cuidado de pacientes con lesión medular, auxiliándolos y contribuyendo en su autonomía, a través de las intervenciones propuestas.

Descriptores: Diagnóstico de enfermería; Traumatismos de la columna vertebral; Cuidados de enfermería

1 Mestre em Ciências da Saúde pelo Departamento de Enfermagem da Universidade Federal de São Paulo - UNIFESP - São Paulo $(S P)$, Brasil, Professora instrutora do Departamento de Medicina Social da Faculdade de Ciências Médicas da Santa Casa de São Paulo - FCMSCSP - São Paulo(SP), Brasil. Mestre em Ciências da Saúde pelo Departamento de Enfermagem da Universidade Federal de São Paulo - UNIFESP - São Paulo(SP), Brasil.

2 Professora Titular do Departamento de Enfermagem da Universidade Federal de São Paulo - UNIFESP - São Paulo(SP), Brasil

3 Professora Assistente da Escola de Enfermagem da Universidade Federal do Rio Grande do Sul - UFRGS - Rio Grande do Sul (RS), Brasil.

4 Professora Assistente do Departamento de Medicina Social da Faculdade de Ciências Médicas da Santa Casa de São Paulo FCMSCSP - São Paulo(SP), Brasil.

5 Doutora em Enfermagem. Professora Adjunto do Departamento de Enfermagem da Universidade Federal de São Paulo - UNIFESP - São Paulo(SP), Brasil 


\section{INTRODUÇÃO}

O contingente de deficientes físicos, especialmente os portadores de lesão raquimedular, é um fato alarmante no mundo atual. No entanto, a possibilidade de tratar fraturas e luxações da coluna se aperfeiçoou durante os últimos 20 ou 30 anos, em parte como resultado do trabalho em equipe de médicos, enfermeiras ${ }^{\text {a }}$, fisioterapeutas e da utilização de técnicas de emergência avançadas ${ }^{(1-2)}$.

Estudos ${ }^{(3)}$ constataram que durante a Primeira Guerra Mundial, devido ao elevado número de indivíduos acometidos por trauma raquimedular, nunca antes visto, foram realizadas grandes observações dos efeitos imediatos de lesões medulares, sendo que $80 \%$ das vítimas morriam nas primeiras semanas em decorrência, principalmente, de infecção em escaras e de cateterização vesical, verificando-se que sobreviviam, em sua maioria, os pacientes com lesões parciais. Para esses autores, as lesões agudas medulares são ocorrências graves e inesperadas, que acometem anualmente milhares de indivíduos de todas as faixas etárias, levando a conseqüências que persistirão por toda a vida.

As repercussões econômicas são igualmente trágicas, pois os custos do tratamento são estimados em 50 mil dólares/paciente no primeiro ano e em 500 mil dólares durante o resto da vida. Todavia, a grande incidência em jovens, com posterior incapacitação para o trabalho, tornam o "prejuízo" incalculável e os gastos com os indivíduos nos programas de reabilitação enormes e, além disso, considera-se a perda da produtividade do indivíduo na comunidade/família.

Estatísticas norte-americanas mostraram uma população de aproximadamente 200 mil indivíduos, com uma incidência anual de aproximadamente 10 mil casos. As ocorrências de sobrevida aumentam a cada dia, no entanto, presume-se que esse número possa ser maior, uma vez que os protocolos de necropsia podem ser falhos na detecção de trauma medular ${ }^{(3)}$.

Durante os últimos 20 anos, nos Estados Unidos persistiram ainda como principais causas, os acidentes automobilísticos $(45 \%)$, as quedas $(22 \%)$, os atos de violência (16\%) e a participação em esportes (13\%). No Brasil, na maioria dos casos, essas lesões são de origem traumática, sendo que as causas externas mais freqüentes são os ferimentos por arma de fogo, seguidos dos acidentes automobilísticos e quedas ${ }^{(1,4-5)}$.

Em função dos recursos existentes atualmente, o aumento da sobrevida destes pacientes é uma realidade, sendo assim importante aos profissionais de saúde, um maior conhecimento sobre o assunto, principalmente sobre as complicações clínicas que são praticamente restritas às vítimas de lesões medulares ${ }^{(3)}$.

a Termo utilizado no feminino devido ao maior número de mulheres na profissão.
Faro $^{(6)}$ comenta que a pessoa com lesão medular apresenta alterações significativas de motricidade e sensibilidade, dentre tantas outras, ocorrendo, muitas vezes, dependência de terceiros para atividades antes tidas como corriqueiras e outras mais íntimas, como a higiene após eliminações. Portanto, esse tipo de paciente é caracterizado como altamente dependente dos cuidados de enfermagem ou de terceiros para atividades básicas da vida diária, como alimentar-se, vestir-se, despir-se, posicionar-se na cama ou na cadeira e higienizar-se.

Considerando-se a gravidade desses casos e a grande dependência dos cuidados de enfermagem que exigem, desenvolveu-se o presente estudo na intenção de aprofundar conhecimentos, capaz de nortear as ações de enfermagem, com a finalidade de prestar uma assistência especializada ao paciente lesado medular, promovendo a recuperação da sua saúde, auxiliando na reabilitação e prevenindo complicações, possibilitando, assim, que o mesmo reassuma sua autonomia, com retorno ao seu ambiente social. Estas ações são preocupações importantes da enfermeira, enquanto elemento da equipe multiprofissional, que auxilia na assistência ao lesado medular.

Para tanto, é fundamental adotar um método de trabalho capaz de direcionar e organizar as atividades de enfermagem de acordo com as necessidades de cada paciente. O processo de enfermagem possibilita esta organização, pois é uma metodologia embasada em referências teóricas, de acordo com a filosofia de cada instituição, podendo atender aos pacientes de forma individualizada $\mathrm{e}$ integral nas suas necessidades biopsicossociais ${ }^{(7-8)}$.

Considerando-se que o diagnóstico de enfermagem é um dos principais desafios das enfermeiras, pois dele dependem os resultados de suas ações, este estudo tem como objetivo identificar os diagnósticos de enfermagem em indivíduos com lesão medular, internados numa unidade de Ortopedia e Traumatologia, de acordo com a proposta da North American Nursing Diagnosis Association (NANDA) e, de maneira subseqüente, apresentar propostas de intervenções de enfermagem, com base na Nursing Interventions Classification (NIC) ${ }^{(9-11)}$.

A utilização das classificações é de extrema importância, pois possibilita uma padronização da linguagem para os diagnósticos, intervenções e resultados de enfermagem, favorecendo a reflexão sobre as habilidades das enfermeiras em examinar as tendências de sua prática e avaliar a qualidade de cuidados prestados aos pacientes.

No que diz respeito ao desenvolvimento da NIC, oito razões para o seu desenvolvimento foram listadas pelo grupo responsável por sua criação: - padronização da nomenclatura dos tratamentos de enfermagem; - expansão do conhecimento de enfermagem sobre os vínculos entre diagnósticos, tratamentos e resultados; 
- desenvolvimento da enfermagem e sistemas informatizados no cuidado à saúde; - ensino da tomada de decisão para estudantes de enfermagem; - determinação dos custos de serviços providos pelas enfermeiras; - planejamento dos recursos necessários nos ambientes de prática de enfermagem; - linguagem para comunicar a função específica da enfermagem; - articulação com os sistemas de classificação de outros profissionais de saúde ${ }^{(10-11)}$.

Atualmente, a Classificação das Intervenções de Enfermagem (NIC) representa uma das mais avançadas propostas em termos de pesquisas sobre intervenções de enfermagem, apresentada numa estrutura validada e codificada, contendo 486 intervenções e mais de $12 \mathrm{mil}$ atividades, articulada com a classificação de diagnósticos de enfermagem da NANDA e a classificação dos resultados de enfermagem - NOC ${ }^{(10-13)}$.

Ressaltando-se os benefícios da linguagem padronizada e acreditando-se que a assistência de enfermagem deva ocorrer de forma sistematizada, visando à excelência da prática da enfermagem, é que se adotou a NANDA e a NIC como referenciais neste estudo, como formas de dar visibilidade ao fazer da profissão.

Por fim, em relação ao cuidado do paciente com lesão medular ressalta-se a opinião de alguns autores, que afirmam que a reabilitação, como processo complexo que é, exige custos para sua implantação e manutenção ${ }^{(14)}$. No entanto, esses custos podem se revelar elevados a curto prazo, sendo a médio e longo prazos justificados pela obtenção da redução da dependência, e conseqüente reintegração social do indivíduo, com a melhora da sua produtividade.

\section{MÉTODOS}

A pesquisa foi desenvolvida num hospital-escola da capital paulista, reconhecido de utilidade pública federal, estadual e municipal. É uma instituição que atua em nível terciário de assistência, com alguns pólos de assistência em nível quaternário, atendendo indivíduos, principalmente através do Sistema Único de Saúde (SUS).

Trata-se de um estudo clínico prospectivo, realizado em pacientes com diagnóstico médico de lesão medular, internados na Unidade de Ortopedia e Traumatologia do referido hospital, no período de janeiro de 2000 a julho de 2002. A unidade é composta de 38 leitos, sendo 15 masculinos e 15 femininos, além de 8 infantis (masculinos e femininos) e, possui sistematização de enfermagem implantada.

A coleta de dados foi realizada após a aprovação do projeto de pesquisa pelo comitê de ética em pesquisa da UNIFESP. Todos os pacientes incluídos na investigação foram orientados quanto ao estudo, aceitando participar do mesmo. Na impossibilidade do consentimento pelo pa- ciente, um responsável por este assumiu o acordo de participação, através do consentimento livre e esclarecido.

A amostra do estudo foi composta de dez pacientes, que constituiu o " $\mathrm{N}$ " total de internações de pacientes com o diagnóstico médico de lesão medular, na unidade pesquisada, no período estudado de dois anos e maio

Os dados foram coletados por meio de um instrumento criado para coleta de dados de enfermagem na unidade, o qual constava de identificação do paciente, anamnese, exame físico geral, especial e ortopédico, e avaliação das necessidades humanas básicas. Este instrumento foi aplicado na admissão dos pacientes na unidade campo de estudo, no período de janeiro de 2000 a julho de 2002.

A seguir, os diagnósticos de enfermagem foram identificados e analisados pelas autoras, sendo que para os de maior freqüência, ou seja, os que tiveram percentual acima de $50 \%$, foi construída uma relação com as suas características definidoras e fatores relacionados ou de risco, para posteriormente propor intervenções, segundo a Nursing Interventions Classification - NIC.

Dessa forma, as intervenções de enfermagem foram propostas pelas autoras, com base nos diagnósticos identificados e na NIC, levando em consideração as características definidoras e os fatores relacionados ou de risco, para posteriormente serem analisadas por duas enfermeiras experts em Ortopedia e Traumatologia. Essas experts possuíam experiência mínima de cinco anos na assistência, ensino e pesquisa nessa área, contribuindo, assim, com suas experiências profissionais e conhecimento específico, na elaboração das propostas de intervenções de enfermagem para cada diagnóstico de enfermagem identificado nos pacientes portadores de lesão medular.

\section{RESULTADOS E DISCUSSÃO}

Num primeiro momento, apresenta-se os dados resultantes do perfil dos pacientes com diagnóstico de lesão medular, internados na enfermaria de Ortopedia e Traumatologia do hospital campo de investigação.

Em relação ao sexo e à idade, o maior número pertence ao sexo masculino (90\%), com idade variando entre 23 e 32 anos (40\%) ou na faixa dos 50 anos ou mais (30\%). Estes dados apresentam similaridade aos estudos que apontam o predomínio de uma população jovem de lesados medulares, na maioria do sexo masculino ${ }^{(1,4-5,15-18)}$. No entanto, a ocorrência de $30 \%$ de pacientes na faixa etária de 50 anos ou mais, levou ao questionamento se isto estaria relacionado ao processo de envelhecimento da população brasileira, com maior expectativa de vida, ou se outros fatores como a violência urbana poderiam ter contribuído para esta estatística.

Observando-se o grau de escolaridade, verificou-se que $50 \%$ dos pacientes têm o nível de $1^{\circ}$ grau, completo 
ou incompleto, $40 \%$ possuem o $2^{\circ}$ grau e apenas um deles (10\%) referiu ser analfabeto. Ressalta-se que nesta amostra não foi encontrado nenhum paciente com $3^{\circ}$ grau. Faro ${ }^{(1)}$ encontrou o mesmo predomínio de grau de instrução.

O estado civil representou $50 \%$ de pacientes casados e $50 \%$ solteiros.

Quanto ao aspecto religioso, prevalece a religião católica em $70 \%$ dos casos, sendo que os demais $30 \%$ referiram pertencer a denominações religiosas evangélicas e, 20\% especificaram serem Testemunhas de Jeová. Estes achados também corroboram os identificados por Faro $^{(1)}$.

Os resultados referentes aos diagnósticos de enfermagem desses pacientes são apresentados na tabela 1 , demonstrando a frequiência absoluta, em ordem decrescente, das 15 categorias diagnósticas de enfermagem identificadas entre os 10 casos estudados.

Tabela 1 - Freqüência das categorias diagnósticas de enfermagem em pacientes portadores de lesão medular internados na unidade de Ortopedia e Traumatologia de um hospital-escola. (São Paulo, 2000-2002)

\begin{tabular}{lcc}
\hline DIAGNÓSTICOS DE ENFERMAGEM & N & $\%$ \\
\hline Mobilidade física prejudicada & 10 & 100 \\
Déficit no autocuidado para banho e higiene & 10 & 100 \\
Déficit no autocuidado para vestir-se e arrumar-se & 10 & 100 \\
Disfunção sexual & 08 & 80 \\
Risco para infecção & 08 & 80 \\
Risco para integridade da pele prejudicada & 07 & 70 \\
Incontinência urinária total & 03 & 30 \\
Constipação & 03 & 30 \\
Ansiedade & 03 & 30 \\
Integridade da pele prejudicada & 03 & 30 \\
Risco para disreflexia autonômica & 03 & 30 \\
Déficit no autocuidado para alimentar-se & 02 & 20 \\
Déficit de conhecimento & 02 & 20 \\
Retenção urinária & 01 & 10 \\
Dor & 01 & 10 \\
\hline
\end{tabular}

Observa-se que os diagnósticos de enfermagem predominantes foram: mobilidade física prejudicada (100\%); déficit de autocuidado para banho e higiene (100\%); déficit de autocuidado para vestir-se e arrumar-se (100\%); disfunção sexual (80\%); risco para infecção (80\%) e risco para integridade da pele prejudicada $(70 \%)$. É possível identificar, neste estudo, outros diagnósticos, tais como: incontinência urinária total; constipação; ansiedade; integridade da pele prejudicada, encontrados em $30 \%$ dos pacientes; risco para disreflexia autonômica; déficit no autocuidado para alimentar-se e déficit de conhecimento, numa freqüência de $20 \%$, e os diagnósticos de retenção urinária e dor, que foram detectados num único paciente $(10 \%)$.

A literatura aponta resultados semelhantes aos resultados apresentados. Faro ${ }^{(1)}$ demonstra que $100 \%$ dos pacientes estudados apresentaram a mobilidade física prejudicada e potencial para prejuízo da integridade da pele. Os outros diagnósticos mais freqüentes foram: déficit no autocuidado para vestir-se arrumar-se $(89,47 \%)$, déficit no autocuidado para banho $(86,84 \%)$ e incontinência urinária $(71,05 \%)$, destacando ainda risco para infecção $(68,42 \%)$ e obstipação percebida $(52,63 \%)$.

No presente estudo, o diagnóstico de mobilidade física prejudicada foi identificado em $100 \%$ dos pacientes e é importante observar que o mesmo está muito relacionado com outros diagnósticos encontrados, como déficit no autocuidado para banho e higiene, déficit no autocuidado para vestir-se/arrumar-se e risco para integridade da pele prejudicada. Na verdade, poder-se-ia dizer que a mobilidade física prejudicada constitui-se num fator relacionado a estes outros diagnósticos, pois pressupõe um prejuízo neuromuscular e musculoesquelético, listado entre os fatores a eles relacionados. Esta interligação deve ser mais profundamente estudada, pois talvez seja possível identificar um perfil sindrômico na associação de diagnósticos de enfermagem deste tipo de pacientes.

No que se refere ao diagnóstico Risco para infecção, $\operatorname{Barros}^{(8)}$ diz que é o diagnóstico de enfermagem mais presente em pacientes hospitalizados, em virtude de fatores diversos do processo de hospitalização, requerendo uma atitude preventiva que deve nortear as ações da enfermagem no plano de cuidado, levando em consideração sua interface com outros diagnósticos.

A seguir são apresentados os 6 diagnósticos de enfermagem mais freqüentes (Tabela 2), descritos com os seguintes componentes: Título (nome do diagnóstico); Características Definidoras - CD; Fatores relacionados - FR (condições que contribuem para a afirmação diagnóstica) e Fatores de Risco - Fr (condições que aumentam a vulnerabilidade).

Tabela 2 - Freqüência das características definidoras do diagnóstico de enfermagem "Mobilidade física prejudicada" e fatores relacionados em pacientes portadores de lesão medular, internados em Unidade de Ortopedia e Traumatologia de um hospital-escola. (São Paulo, 20002002)

\begin{tabular}{lrr}
\hline $\begin{array}{l}\text { CARACTERÍSTICAS DEFINIDORAS/ } \\
\text { FATORES RELACIONADOS }\end{array}$ & N & $\%$ \\
\hline CD: Capacidade limitada para desempenhar as & 10 & 100 \\
habilidades motoras grossas e finas & 10 & 100 \\
FR: Prejuízos sensorioperceptivos & 10 & 100 \\
FR: Prejuízos neuromusculares & 10 & 100 \\
FR: Força e resistência diminuída & 10 & 100 \\
FR: Força, controle e massa muscular diminuídos & 10 & 100 \\
FR: Dor & 01 & 10 \\
\hline
\end{tabular}


Nota-se, na tabela 2, que, no diagnóstico Mobilidade física prejudicada houve um predomínio de $100 \%$ da característica definidora capacidade limitada para desempenhar habilidades motoras grossas e finas. Os fatores relacionados de maior incidência foram os seguintes: prejuízos sensorioperceptivos (100\%), prejuízos neuromusculares $(100 \%)$, força e resistência diminuída (100\%) e força, controle e massa muscular diminuídos $(100 \%)$. O fator relacionado dor foi encontrado em apenas um paciente $(10 \%)$.

Tabela 3 - Freqüência das características definidoras do diagnóstico de enfermagem "Déficit no autocuidado: banho e higiene" e fatores relacionados em pacientes portadores de lesão medular, internados em Unidade de Ortopedia e Traumatologia de um hospital-escola. (São Paulo, 2000-2002)

\begin{tabular}{lcr}
\hline $\begin{array}{c}\text { CARACTERÍSTICAS DEFINIDORAS/ } \\
\text { FATORES RELACIONADOS }\end{array}$ & N & $\%$ \\
\hline CD: Incapacidade de chegar à fonte de água & 10 & 100 \\
CD: Incapacidade de lavar o corpo ou parte do & 10 & 100 \\
corpo & & \\
CD: Incapacidade de pegar artigos para banho & 02 & 20 \\
FR : Prejuízo neuromuscular & 10 & 100 \\
FR : Prejuízo musculoesquelético & 10 & 100
\end{tabular}

Na tabela 3 observa-se que a maior incidência das características definidoras para o diagnóstico de Déficit no autocuidado para banho e higiene foi na: incapacidade de chegar à fonte de água $(100 \%)$ e incapacidade de lavar o corpo ou partes do corpo (100\%). A característica definidora que obteve a menor incidência (20\%) foi a incapacidade de pegar artigos para banho. Com relação aos fatores relacionados, encontrou-se prejuízo neuromuscular (100\%), prejuízo musculoesquelético $(100 \%)$.

Tabela 4 - Freqüência das características definidoras do diagnóstico de enfermagem "Déficit no autocuidado: vestir-se/arrumar-se" e fatores relacionados em pacientes portadores de lesão medular, internados em Unidade de Ortopedia e Traumatologia de um hospital-escola. (São Paulo, 2000-2002)

\begin{tabular}{lrr}
\hline $\begin{array}{c}\text { CARACTERÍSTICAS DEFINIDORAS/ } \\
\text { FATORES RELACIONADOS }\end{array}$ & N & $\%$ \\
\hline $\begin{array}{c}\text { CD: Capacidade prejudicada de por/tirar/fechar/ } \\
\text { obter roupas }\end{array}$ & 10 & 100 \\
CD: Incapacidade de colocar roupas na parte & 07 & 100 \\
$\quad$ superior do corpo & & 20 \\
FR : Prejuízo neuromuscular & 10 & 100 \\
FR : Prejuízo musculoesquelético & 10 & 100 \\
FR : Prejuízo perceptivo ou cognitivo & 10 & 100 \\
FR : Dor & 01 & 10 \\
FR : Ansiedade grave & 01 & 10 \\
\hline
\end{tabular}

A tabela 4 demonstra que a maior incidência (100\%) das características definidoras para o diagnóstico de Déficit no autocuidado para vestir-se/arrumar-se foi a capacidade prejudicada de pôr ou tirar itens necessários do vestuário, fechar roupas, obter ou repor artigos de vestuário e $70 \%$ para incapacidade de colocar roupas na parte superior do corpo. Quanto aos fatores relacionados, encontrou-se prejuízo neuromuscular, prejuízo musculoesquelético e prejuízo perceptivo ou cognitivo em $100 \%$ dos pacientes, além de dor e ansiedade grave em $10 \%$ dos mesmos.

Tabela 5 - Características definidoras do diagnóstico de enfermagem "Disfunção sexual" e fatores relacionados encontrado nos pacientes portadores de lesão medular, internados em Unidade de Ortopedia e Traumatologia de um hospital-escola. (São Paulo, 2000-2002)

\begin{tabular}{lrr}
\hline $\begin{array}{c}\text { CARACTERÍSTICAS DEFINIDORAS/ } \\
\text { FATORES RELACIONADOS }\end{array}$ & N & $\%$ \\
\hline $\begin{array}{c}\text { CD: Capacidade prejudicada de por/tirar/fechar/ } \\
\text { obter roupas }\end{array}$ & 10 & 100 \\
CD: Incapacidade de colocar roupas na parte & 07 & 100 \\
$\quad$ superior do corpo & & 20 \\
FR : Prejuízo neuromuscular & 10 & 100 \\
FR : Prejuízo musculoesquelético & 10 & 100 \\
FR : Prejuízo perceptivo ou cognitivo & 10 & 100 \\
FR : Dor & 01 & 10 \\
FR : Ansiedade grave & 01 & 10 \\
\hline
\end{tabular}

O diagnóstico de enfermagem de Disfunção sexual foi encontrado em $80 \%$ dos pacientes, e a tabela 5 indica que $100 \%$ dos pacientes apresentaram as características definidoras: incapacidade de alcançar a satisfação desejada e verbalização do problema, $50 \%$ apresentavam limitações reais ou percebidas impostas pela doença e/ou pela terapêutica e $25 \%$ alteração no relacionamento com a pessoa significativa. O que fundamentou a formulação desse diagnóstico foi o relato dos pacientes quanto à inatividade sexual.

Na esfera da sexualidade, a NANDA propõe dois diagnósticos: Disfunção sexual e Padrões de sexualidade alterados. Baseando-se nas características dos dois diagnósticos e considerando o estudo de Faro ${ }^{(1)}$, acreditou-se que o diagnóstico de disfunção sexual é o mais apropriado para este tipo de paciente.

Os achados neste estudo coincidem com o de Faro(1), no tocante à disfunção sexual detectou-se, por meio do depoimento dos participantes, que a maioria apresentava ereção reflexa ou psicogênica e a ejaculação estava ausente na maioria. Para esta autora, a disfunção sexual está relacionada ao nível e grau da lesão e, ainda com o intuito de esgotar os aspectos da disfunção erétil, esclarece que tanto a ereção reflexa como a psicogênica, muitas vezes não são suficientemente duradouras e consistentes para permitir a penetração vaginal. 
Os pacientes relatam que têm medo de voltar a ter relações sexuais após o trauma, com receio de impotência ou de gravidez. O que reforça nosso pensar quanto à necessidade de compreensão e orientação dessa clientela.

Tabela 6 - Fatores de risco relacionados do diagnóstico de enfermagem "Risco para infecção" encontrado nos pacientes portadores de lesão medular, internados em Unidade de Ortopedia e Traumatologia de um hospitalescola. (São Paulo, 2000-2002)

\begin{tabular}{lcc}
\hline FATORES DE RISCO & N & $\%$ \\
\hline Exposição ambiental a patógenos aumentada & 08 & 100 \\
Procedimentos invasivos & 08 & 100 \\
Destruição de tecidos e exposição ambiental & 03 & 37,5 \\
aumentada & 03 & 37,5 \\
Defesas primárias inadequadas & 03 \\
\hline
\end{tabular}

O diagnóstico de Risco para infecção foi encontrado em $80 \%$ dos casos e, como fatores de risco, destaca-se a exposição ambiental a patógenos aumentada devido ao período de internação (100\%) e procedimentos invasivos $(100 \%)$, relacionados aos procedimentos realizados no período de internação como a punção venosa e cateterismo vesical intermitente. Foi encontrado em $37,5 \%$ da amostra a destruição de tecidos e exposição ambiental aumentada, relacionados a incisões cirúrgicas e úlceras de pressão e em outros $37,5 \%$ observaram-se defesas primárias inadequadas, associadas à pele rompida e peristalse alterada.

Tabela 7 - Fatores de risco do diagnóstico de enfermagem "Risco para integridade da pele prejudicada" encontrado nos pacientes portadores de lesão medular, internados em Unidade de Ortopedia e Traumatologia de um hospital-escola. (São Paulo, 2000-2002)

\begin{tabular}{lcc}
\hline FATORES DE RISCO & N & $\%$ \\
\hline Imobilização física & 07 & 100 \\
Proeminências esqueléticas & 07 & 100 \\
Sensibilidade alterada & 07 & 100 \\
\hline
\end{tabular}

Na tabela 7 verifica-se que, para o diagnóstico de enfermagem Risco para a integridade da pele prejudi$c a d a$, os fatores de risco: imobilização física; proeminências esqueléticas e sensibilidade alterada foram encontrados em todos os pacientes (100\%).

Após a identificação de todos diagnósticos de enfermagem realizou-se o levantamento das suas respectivas intervenções de enfermagem, baseadas na NIC. Posteriormente, as intervenções de enfermagem foram analisadas, através do julgamento terapêutico realizado por enfermeiras experts, em concordância com as autoras do estudo.
Os resultados desta análise levaram à apresentação de diversas propostas para as resoluções dos problemas encontrados e foram sugeridas atividades para as intervenções apresentadas.

Assim, as 26 intervenções da NIC apontadas para os diagnósticos de enfermagem mais freqüientemente indicados para o paciente com lesão medular são as seguintes:

- Terapia com exercícios: deambulação;

- Terapia com exercícios: mobilidade articular;

- Posicionamento;

- Banho;

- Assistência no autocuidado: banho e higiene;

- Vestir;

- Cuidados com os cabelos;

- Assistência no autocuidado: vestir-se/arrumar-se;

- Aconselhamento sexual;

- Controle de infecção;

- Proteção contra infecção;

- Controle de pressão sobre áreas do corpo;

- Prevenção de úlcera de pressão;

- Cuidados na incontinência urinária;

- Controle da constipação/impactação;

- Redução da ansiedade;

- Cuidados com local de incisão;

- Supervisão da pele;

- Cuidados com lesões;

- Controle da disreflexia;

- Alimentação;

- Assistência no autocuidado: alimentação;

- Ensino: processo da doença;

- Cateterização vesical;

- Cuidados na retenção urinária;

- Controle da dor.

\section{CONCLUSÕES}

Os resultados encontrados permitiram concluir que os pacientes portadores de lesão medular apresentam alguns diagnósticos de enfermagem específicos, demonstrando a necessidade de um plano de cuidados próprios para este tipo de paciente, abordando de forma ampla o indivíduo jovem, no auge da sua capacidade produtiva.

Dessa forma, cabe à enfermagem utilizar recursos que possam aprimorar a assistência prestada a esses pacientes. A implementação dos diagnósticos e intervenções de enfermagem, de maneira precisa, constituem uma alternativa a ser utilizada, possibilitando a melhoria da qualidade dos resultados esperados. Portanto, é oportuno lembrar a necessidade do preparo da enfermeira, bem como dos familiares que participam do processo de assistência ao lesado medular, sendo necessárias a realização de estudos, orientações e treinamentos neste âmbito do cuidado. 
Como já destacado anteriormente, as intervenções de enfermagem a serem realizadas para os diagnósticos de enfermagem encontrados foram eleitas a partir da NIC, através do julgamento terapêutico realizado por duas enfermeiras experts em Ortopedia e Traumatologia, em concordância com as autoras. Todavia, é importante salientar a necessidade de utilizá-las na prática clínica, com as suas respectivas atividades, como forma de validálas em futuras pesquisas.

Uma das principais limitações deste estudo refere-se à amostra, devido ao pequeno número de internações no período estudado, visto que o hospital campo de pesquisa deixou de ser referência para o atendimento ao lesado medular. No entanto, apesar de suas limitações, esperase poder ter contribuído com as enfermeiras que cuidam do paciente com lesão medular, oferecendo-lhes a possibilidade de aprimorar seu conhecimento na área, através da proposta de intervenções para os diagnósticos de enfermagem mais freqüentes, auxiliando-as a qualificar a assistência prestada.

Pensa-se, ainda, ter contribuído para a reflexão sobre a complexa problemática do lesado medular, como também ter servido de estímulo para a realização de outras pesquisas, que possam evidenciar diferentes resultados e, assim, possibilitar a elaboração de novas propostas de intervenções para estes pacientes tão necessitados de cuidado.

\section{REFERÊNCIAS}

1. Faro ACM. Estudo das alterações da função sexual em homens paraplégicos [tese]. São Paulo: Escola de Enfermagem da Universidade de São Paulo; 1991.

2. Barros Filho TEP. Avaliação padronizadas nos traumatismos raquimedulares. Rev Bras Ortop. 1994;29(3):99-106.

3. Gondim FAA, Oliveira GR, Rôla FH. Complicações clínicas de injúrias medulares. Rev Bras Neurol. 1998;34(2):47-54.

4. Spósito MMM, Laredo Filho J, Braga FM, Novo NF. Paraplegia por lesão medular: estudo epidemiológico em pacientes atendidos para reabilitação. Rev Paul Med. 1986;104(4): 196-202.

5. Santos LCR. Lesão traumática da medula espinhal: estudo retrospectivo de pacientes internados no Instituto de Ortopedia e Traumatologia do Hospital das Clínicas da Faculdade de Medicina da Universidade de São Paulo entre 1982 - 1987 [tese]. São Paulo: Escola de Enfermagem, Universidade de São Paulo; 1989.

6. Faro ACM. Fatores de risco para úlcera de pressão: subsídios para prevenção. Rev Esc Enferm USP. 1999;33 (3):279-83.

7. Rothrock JC, Meeker MH. Alexander: cuidados de enfermagem ao paciente cirúrgico. 10 ed. Rio de Janeiro: Guanabara Koogan; 1997.
8. Barros ALBL. O trabalho docente assistencial de enfermagem no Hospital São Paulo da UNIFESP/EPM [tese]. São Paulo: Departamento de Enfermagem, Escola Paulista de Medicina da UNIFESP da Universidade Federal de São Paulo; 1998.

9. North American Nursing Diagnosis Association (NANDA). Diagnósticos de enfermagem da NANDA : definições e classificação 2001-2002. Traduzido por: de Michel J. L. M. Porto Alegre: Artmed; 2002.

10. McCloskey JC, Bulechek GM. Nursing interventions classification (NIC). 3rd ed. St. Louis: Mosby; 2000.

11. McCloskey J, Bulechek GM. Classificação das intervenções de enfermagem - NIC. 3a ed. Porto Alegre: Artmed; 2004.

12. Johnson M, Maas M, Moorhead S. Classificação dos resultados de enfermagem - NOC. 2a ed. Porto Alegre: Artmed; 2004.

13. Johnson M, Bulechek G, Dochterman Mccloskey J, Maas M, Moorhead S. Nursing diagnoses, outcomes, and interventions: NANDA, NOC and NIC likages. St. Louis: Mosby; 2001.

14. Santos VLCG, Miyadahira, AMK, Kimura M. Assistência em reabilitação: um processo marcado por deficiências. Rev Gaúch Enferm. 1990;11(2):5-11.

15. Crigler L. Sexual concerns of the spinal cord-injured. Nurs Clin North Am. 1974;9 (4):703-16.

16. Bogossian L. Traumatismo em geral: estudo etiopatológico dos traumas mecânicos. J Bras Med. 1981;41(2):61-70.

17. Faro ACM, Higuchi CM. Atuação de enfermagem em um pronto socorro de ortopedia e traumatologia. Rev Esc Enferm USP. 1987;21(2):161-79.

18. Faro ACM. Do diagnóstico à conduta de enfermagem: a trajetória na reabilitação do lesado medular [tese]. São Paulo: Escola de Enfermagem da Universidade de São Paulo; 1998. 